\title{
Correction to: Bone graft materials for posterolateral fusion made simple: a systematic review
}

\author{
Matthew T. Morris ${ }^{1} \cdot$ Sandip P. Tarpada ${ }^{1} \cdot$ Woojin Cho ${ }^{1,2}$
}

Published online: 7 July 2021

○) Springer-Verlag GmbH Germany, part of Springer Nature 2021

\section{Correction to: European Spine Journal (2018) 27:1856-1867 https://doi.org/10.1007/s00586-018-5511-6}

The original article been published with some corrections in text and in Table 6. The details of the corrections are given below.

The Results section should be corrected to the following (corrections are in bold):

Thirty six studies looked at ICBG in conjunction with LAG (29), APC (2), DBM (1), BMPs (3), or BCP(1). Mean fusion rates among these ranged from $68 \%$ to $91.5 \%$. Twenty-two studies (and twenty-three study groups) evaluated fusion rates of LAG, either isolated (4) or combined with APC (1), DBM (4), BMA (4), BMP (1), allograft (1), or ceramic extenders (8). Of these, 19 (82.6\%) study groups observed solid fusion in greater than 80 percent of patients by 1-year follow-up. Five papers focused on BMA.
1 in conjunction with allograft, 1 with DBM, and 3 with ceramic carriers. Of these, 2 showed fusion rates above $80 \%$. Finally, a total of $\mathbf{1 7}$ papers involved evaluation of BMPs. Of these, 12 studies looked at rhBMP-2, and 100\% observed successful fusion rates of at least $80 \%$ at 1 year. Five studies looked at rhBMP-7 and collagen, and none showed fusion rates above $80 \%$ [Table 6].

The Bone Marrow Aspirate/Concentrate section of the Discussion should be corrected as follows

More uniformly positive results have been observed when LAG is added to this graft mix, with $\mathbf{4}$ of $\mathbf{4}$ studies reporting fusion rates $\geq 80 \%$ and $\mathbf{1}$ of $\mathbf{1}(100 \%)$ similar to autograft control. $^{2}$

An evidence table for the included studies has been added as electronic supplementary material.
The original article can be found online at https://doi.org/10.1007/ s00586-021-06900-6.

\footnotetext{
Woojin Cho

woojinchomd@aol.com

1 Department of Orthopaedic Surgery, Albert Einstein College of Medicine, Bronx, NY, USA

2 Department of Orthopaedic Surgery, Montefiore Medical Center, 3400 Bainbridge Avenue, Bronx, NY 10467-2404, USA
} 
Table 6 Summary of search results (corrected)

\begin{tabular}{lllll}
\hline Graft type & $\begin{array}{l}\text { Number of } \\
\text { studies }\end{array}$ & Mean fusion rate & Fusion rate range & Standard deviation \\
\hline ICBG (+LAG) & 29 & $77.29 \%$ & $40-100 \%$ & $+/-16.1 \%$ \\
ICBG+APC & 2 & $68 \%$ & $62-74 \%$ & $+/-6.0 \%$ \\
ICBG+DBM & 1 & $70 \%$ & $70 \%$ & - \\
ICBG+BMP & 3 & $78.6 \%$ & $69-97 \%$ & $+/-15.9 \%$ \\
ICBG+ceramics & 1 & $91.5 \%$ & $91.5 \%$ & - \\
LAG & 4 & $80 \%$ & $65-100 \%$ & $+/-14.7 \%$ \\
LAG+APC & 1 & $75 \%$ & $75 \%$ & - \\
LAG+DBM & 4 & $78.93 \%$ & $60-87 \%$ & $+/-14.4 \%$ \\
LAG+BMA & 4 & $91.68 \%$ & $85-95 \%$ & $+/-4.2 \%$ \\
LAG+BMP & 1 & $80 \%$ & $80 \%$ & - \\
LAG+allograft & 1 & $93.7 \%$ & $93.7 \%$ & $+/-6.72 \%$ \\
LAG+ceramics & 8 & $89 \%$ & $80-100 \%$ & - \\
BMA+allograft & 1 & $80 \%$ & $80 \%$ & - \\
BMA+DBM & 1 & $70 \%$ & $70 \%$ & $+/-9.84 \%$ \\
BMA+ceramics & 3 & $56.83 \%$ & $5-89 \%$ & $+/-16.0 \%$ \\
BMP+ceramics & 13 & $84.14 \%$ & $55-100 \%$ & $+/-4.0 \%$ \\
BAG & 2 & $84 \%$ & $80-88 \%$ & - \\
Allograft & 1 & $40 \%$ & $40 \%$ & \\
\hline
\end{tabular}

Supplementary Information The online version contains supplementary material available at https://doi.org/10.1007/s00586-021-06900-6.
Publisher's Note Springer Nature remains neutral with regard to jurisdictional claims in published maps and institutional affiliations. 